https://doi.org/10.21670/ref.1912033

Artículos

Recibido el 3 de abril de 2019.

Aceptado el 9 de octubre de 2019.

Publicado el 28 de octubre de 2019.

*Autor para correspondencia: Florencia Costantini, correo electrónico, flor.costantini@hotmail. com.ar

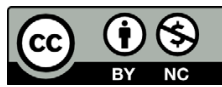

Esta obra está protegida bajo una Licencia Creative Commons Atribución-NoComercial 4.0 Internacional.

\section{Capitales británicos y producción agraria en espacios de frontera. Bahía Blanca, Argentina (1860-1900)}

\author{
British capitals and agrarian production in border \\ areas. Bahía Blanca, Argentina (1860-1900)
}

Florencia Costantinia* (D) https://orcid.org/0000-0001-8669-6851

a Universidad Nacional del Sur, Centro de Estudios Regionales "Profesor Felix Weinberg", Departamento de Humanidades, Bahía Blanca, Provincia de Buenos Aires, Argentina, correo electrónico: flor.costantini@hotmail.com.ar

\title{
Resumen:
}

Con el objetivo de problematizar el proceso modernizador del sur bonaerense argentino, el siguiente artículo investiga el rol que tuvieron las inversiones británicas en la economía rural en el partido de Bahía Blanca entre 1860 y 1900. La metodología empleada es microanalítica y se vale de herramientas provenientes del estudio hermenéutico de documentos, reconstrucción de trayectorias personales y del análisis estadístico. Abordados en su dimensión diacrónica podemos detectar dos modos de intervención del capital británico en la región que se suceden en el tiempo y que fueron acompañados por formas particulares de inmigración: la inversión en estancias y la presencia de compañías legalmente autónomas. Aunque de modo diferencial, ambos momentos propiciaron la producción agropecuaria a gran escala acelerando el corrimiento de la frontera productiva con impactos en los grupos sociales locales como los comerciantes internos de frutos del país y cereales.

Palabras clave: Inversiones británicas, Bahía Blanca, frontera agraria, empresas.

\section{Abstract:}

In order to problematize the modernizing process in the south of Buenos Aires, in the following article we inquired about the role of British investments in the rural economy in Bahía Blanca between 1860 and 1900. The methodology used is microanalytical and uses tools from the hermeneutical study of documents, reconstruction of personal trajectories and statistical analysis. Addressed in its diachronic dimension, we can detect two types of British capital presence in the re-

CÓMO CITAR: Costantini, F. (2019). Capitales británicos y producción agraria en espacios de frontera. Bahía Blanca, Argentina (18601900). [British capitals and agrarian production in border areas. Bahía Blanca, Argentina (1860-1900)]. Estudios Fronterizos, 20 , e033. doi:https://doi.org/10.21670/ref.1912033 
gion that follow one another in time and that were accompanied by particular forms of immigration: the investment of individuals in farms and the presence of free-standing companies. Although differentially, both moments propitiated large-scale agricultural production accelerating the shift of the productive frontier with effects on the conformation of social groups such as the internal traders of the country's fruits and cereals.

Keywords: British investments, Bahía Blanca, agrarian frontier, Business.

\section{Introducción}

Hacia mediados del siglo xIx la disponibilidad de capitales y mercancías en las plazas europeas consolidaron flujos comerciales y financieros hacia otras partes del globo. Su radicación en el territorio americano fue acompañada por la conformación de aparatos estatales modernos y su inserción en este mercado internacional. La articulación de estos factores (inversiones extranjeras-Estado) propició diversas situaciones en los espacios regionales nacionales. En el caso argentino la zona litoral/pampeana se configuró a lo largo del siglo xix en la base de un esquema agroexportador que encontraba en la venta de efectos agrarios su actividad dinamizadora. Aunque el sur de la provincia de Buenos Aires se encuadraba en ella, su inserción se efectuó de manera tardía durante las últimas décadas del novecientos dado el emplazamiento de parcialidades indígenas y las dificultades en las comunicaciones.

Con el fin de abordar la penetración del capital extranjero en este espacio de frontera, el presente artículo analiza las formas de inserción de inversiones británicas en la esfera rural productiva del partido de Bahía Blanca al sur de la provincia de Buenos Aires (Argentina) durante las últimas décadas del siglo xıx. ${ }^{1}$ La periodización elegida responde a los ritmos propios de la pesquisa iniciándose hacia 1860 al asentarse la colonia inglesa, hasta 1900 con la efectiva expansión del ramal férreo de Bahía Blanca a Neuquén.

Nuestra hipótesis sostiene que el capital británico tuvo dos modalidades de intervención para impulsar la producción rural a gran escala en la región: a través de la inversión de particulares y de compañías legamente autónomas (Free Standing Companies). En un primer momento llevada a cabo por los mismos británicos para en un segundo fomentarla a través de los impactos derivados de la extensión de los transportes y la infraestructura. Tanto la primera forma como la segunda posibilitaron, aunque de manera disímil, la transferencia de tecnología, saberes y gestión empresarial que complejizó, a su vez, la plaza bahiense de comercialización. El desempeño de las compañías coadyuvó, como parte de sus estrategias gerenciales y necesidades económicas, a la consolidación y estrechamiento de lazos con el gremio de los comercializadores internos en Bahía Blanca con el fin de asegurar las ventajas que detentaban sobre los transportes (ferrocarriles y puerto).

\footnotetext{
${ }^{1}$ La delimitación corresponde al partido de Bahía Blanca de 1865 que abarcó al oeste el Río Sauce Chico, al sur la bahía de Bahía Blanca, al Norte las sierras de la Ventana y el Río Sauce Grande al este (Goyena, 1892). Esta región semiárida se encuentra en la llanura pampeana en la transición entre la pampa y la Patagonia y por lo tanto se halla en el cruce de tres ecorregiones: pampeana, espinal y monte (Benedetti et al., 2015).
} 
Ante una economía-mundo en expansión, la perspectiva espacial en clave fronteriza permite atender a las fases, ritmos y a los mecanismos que interactuaron a la hora de articular los procesos de acumulación a escala mundial, los nuevos recursos en disputa y los actores que intervinieron en ello. En la expansión decimonónica de la frontera agropecuaria, entendida como los procesos de valorización/colonización de áreas con el fin de desarrollar actividades agrarias en el marco de desarrollo capitalista (Benedetti y Salizzi, 2014), se conjugaron una serie de sujetos e intereses que ligaron la acción estatal con la de agentes económicos, así como también vincularon procesos globales con los locales. Aquí buscamos desentrañar esta trama de confluencias en un espacio específico, considerando que la reestructuración del territorio conjugó, a la vez que redefinió, alianzas y estrategias en los actores, conflictos y resistencias (Salizzi, 2017). En este marco, la indagación sobre la gestión estatal del espacio (Schiavoni, 1997) como los posicionamientos de los sujetos del lugar podría iluminar sobre el sistema de relaciones (Reboratti, 1990) y de continuidades y rupturas que caracterizan esta realidad con dinámica propia (Benedetti y Salizzi, 2014) que lejos de constituirse en un proceso inevitable fue producto de luchas y acuerdos sobre los recursos y el territorio. De este modo, las variables que tendremos en cuenta para el desarrollo de la investigación serán: las modalidades de intervención del capital británico en particular, la evolución en la producción agraria y los actores socio-políticos involucrados.

Si bien en el periodo de estudio la frontera productiva se solapó con la interétnica y corrió en paralelo con la ampliación del dominio territorial del Estado argentino (Benedetti y Salizzi, 2014), el presente trabajo se centra sobre la injerencia del capital británico en la expansión de la frontera productiva aunque no podemos soslayar su imbricación con los demás procesos, materializados por una serie de actores e imaginarios que transitaron la región. ${ }^{2}$ Así pues, a inicios del periodo de estudio, el reciente partido de Bahía Blanca se constituía en un espacio de contacto entre diferentes modos de utilizar, poblar y poner en producción el territorio los cuales implicaban sistemas de relaciones sociales y económicas tendientes a producir y reproducir bienes (Reboratti, 1990). Esta situación fue resultado de la interacción entre, por un lado, los grupos indígenas preexistentes y, por el otro, el proyecto de crecimiento económico capitalista iniciado en el siglo xix y orientado hacia el mercado mundial (con la consecuente acción de los estado(s) provincial y nacional). Esta dinámica fronteriza combinó y transformó las formas de producción e intercambios, las redes de circulación económica, las relaciones de producción en general y las prácticas, valores, creencias e ideas de los grupos involucrados (Mandrini, 2003). La expansión de nuevas formas de producción, asociadas principalmente a la modernidad ganadera y agrícola, por su parte, se interrelacionaron con procesos de concentración capitalista a escalas mundial y nacional que forjaron inéditas interacciones entre capital, trabajo y territorio (Barsky, 1988; Harvey, 2007).

De este modo, si bien la dimensión económica de este proceso es insoslayable, su abordaje no puede desestimar los fenómenos sociales y políticos que los articularon y al entramado social que lo sustentó, en donde empresas y empresarios emergieron como actores importantes en el contexto de expansión capitalista (Reguera, 2009). En tanto la región analizada se encontró bajo la tutela administrativa del Estado, el papel del mismo

\footnotetext{
${ }^{2}$ La vinculación entre estos tres procesos reconoce un correlato simbólico y discursivo que operó a modo de legitimación del avance sobre el territorio por parte de los sectores dominantes nacionales (véase Jiménez y Alioto, 2007; Villar, 2012).
} 
constituye una dimensión a considerar el cual nos remite a las relaciones de poder y las disputas en torno al dominio y la reproducción socio-económica (Bourdieu, 2002).

El abordaje de las relaciones económicas entre Gran Bretaña y Latinoamérica reconoce una amplia bibliografía tendiente a desentrañar sus principales variables (Lluch y Barbero, 2015; Lanciotti y Lluch, 2009; Vázquez y Dalla, 2005). Para el caso argentino el periodo trabajado con mayor profundidad corresponde al de 1870-1914 (Ferns, 1966; Marichal, 1995; Regalsky, 1986), sin embargo actualmente una serie de investigaciones amplían y complejizan este marco temporal (Kulfas, Porta y Ramos, 2002; Lanciotti y Lluch, 2011; Lluch y Lanciotti, 2012). Desde las primeras aproximaciones en clave estructuralista hasta las recientes aportaciones de la historia de las empresas y la internacional business, la problemática de las inversiones extranjeras se ha visto extendida advirtiendo modalidades y ciclos de inversión, superando los antiguos dualismos intereses nacionales/extranjeros, agrarios/industriales y reparando en temáticas tales como la formación del empresariado, las propiedades de organización de las empresas, el rol del Estado, el gremialismo y las empresas públicas (Lluch y Lanciotti, 2012; Lanciotti y Lluch, 2018a; 2018b; Míguez, 1985; Miller, 2015; Regalsky, 1999; Sourrouille, Gatto y Kosacoff, 1984). Estos estudios fueron acompañados por nuevos planteamientos teórico-metodológicos que tensionan agencia y estructura. Estas aproximaciones ponen de relieve la construcción social de los mercados, las redes sociales que lo forman, la normativa y las estrategias en el estudio del devenir económico nacional (Barbero y Rocchi, 2004).

En la región estudiada, la problemática fue abordada preferentemente en clave migratoria (Caviglia, 1984; Cernadas, Buffa y Eberle, 1992; Iglesias, 1968; Monacci, 1979) y desde los abordajes culturales, imaginarios y construcciones simbólicas sobre la ciudad (Ribas, 2008). Tanto Lázzaro (1992) con su análisis del sistema portuario nacional, como Guerreiro (2011) y Miravalles (2013) se abocaron a las empresas británicas de ferrocarril y sus nexos con los trabajadores y el Estado. Rojas (1984) y Cernadas (1992) analizaron el desenvolvimiento de la colonia británica, su rol defensivo y sus conexiones con el poblado de Bahía Blanca. La influencia del capital británico en la economía regional fue remarcada por las investigaciones que analizaron el curso económico y la estructura productiva local como las de Silva (1987), Cernadas (1992), Crisafulli (1994), Viego (2004) y Costantini y Heredia (2018).

Con el propósito de atender a los impactos del capital británico en su dimensión rural, ensayamos una reducción de la escala de análisis que permita examinar las variables desde una región específica. Para interpretar dichos procesos utilizamos un abordaje que contemple las trayectorias personales, el análisis de contenido y cuantitativos de series estadísticas. Como fuentes disponemos de la prensa del periodo, censos y estadísticas estatales, guías y anuarios comerciales. El fin es utilizarlas para construir las diversas estrategias de los agentes detectados y sus repercusiones en el territorio y la producción.

\section{Las inversiones extranjeras y la región en el escenario nacional y provincial}

Desde 1860 el ingreso regular de capitales extranjeros se inició al territorio nacional, su desarrollo se mostró fluctuante pero constante, acelerándose en ciertas coyunturas 
que conjugaron condiciones endógenas y externas propicias (en la década de los años sesenta y la de los ochenta por ejemplo). Esta entrada respondió a los procesos de acumulación que exigían nuevas posibilidades de inversión para garantizar la reproducción del sistema. Con escasos márgenes de rentabilidad en sus propios dominios, los inversores europeos comenzaron a buscarlos en nuevos espacios del globo. Su introducción, asimismo, tuvo una doble función: permitió ampliar la producción exportable de los países emisores de capital y unificó los mercados orientados al exterior (Lluch y Barbero, 2015; Regalsky, 1986).

A través de las inversiones, de las acciones de sus representantes y de la inmigración, el capital británico fue afianzándose y dejando huellas en el territorio nacional. Desarrollando, asimismo, aspectos de la economía funcionales a sus demandas y réditos. Los tipos de inversión pueden diferenciarse en inversiones de particulares, los empréstitos públicos y la acción a través de las free standing companies, cuyas vinculaciones llegaron a ser estrechas (Martorell, 1969; Schvarzer, Regalsky y Gomez, 2007). Desde una perspectiva regional, como es la que se adopta en esta investigación, focalizamos en el primer y último tipo.

Esta nueva dinámica económica resultó posible dadas las facilidades impuestas desde los entes públicos los cuales experimentaron procesos de centralización y complejización. En cuanto a los grupos que vehiculizaron estos procesos, en la segunda mitad del siglo xix se fue conformando un sector dominante compuesto por y con interrelaciones con los terratenientes pampeanos (Sábato, 1991). Fuertemente articulados a ellos, los sectores económicos impulsores de los procesos de acumulación fueron hacendados e inversores en capital fijo e infraestructura, ramas que se alimentaban recíprocamente en una economía de escala crecientemente mundial, en la cual Gran Bretaña tuvo un peso decisivo (Lewis, 2007).

En el marco de expansión capitalista, la necesaria reorganización productiva luego del quiebre de los vínculos coloniales, sumado a la conexión atlántica y su posición estratégica, hizo que el territorio de análisis suscitara la atención del Estado provincial. Ello derivó en la instalación de un fortín de avanzada contra la población indígena: la Fortaleza Protectora Argentina en 1828. Este hecho inauguró un proceso de poblamiento con intentos de colonización que fueron consolidando novedosas relaciones de poder amalgamadas a través de un nuevo modo de producción. A pesar de encontrarse en la zona nodal del incipiente esquema productivo, el sur bonaerense permaneció durante buena parte del siglo xIX con un desarrollo marginal para la nueva estructuración económica que buscaba insertarse en el comercio internacional. ${ }^{3}$ Los pequeños núcleos de población se incorporaron a redes comerciales tanto con las poblaciones indígenas como entre sí (Alioto, 2011; Ratto y Santilli, 2004; Ratto, 2008; Villar y Ratto, 2004).

\section{Un primer modo de presencia en la región: La inversión en estancias}

Hacia la década de 1860 en la región de Bahía Blanca se observa una reorientación productiva a la actividad agropecuaria perdiendo el comercio su importancia relativa.

\footnotetext{
${ }^{3}$ Sin embargo esta situación no impidió, como veremos a lo largo del presente artículo, que se fueran tejiendo los hilos para posibilitar el avance de la frontera productiva a la luz de la conformación del mercado mundial y la estructuración del Estado que viabilizó dicho proceso.
} 
Este fenómeno se debió a los sucesivos proyectos de colonización y a un retroceso en la conflictividad interétnica que, de todos modos, sería provisoria (Ratto y Santilli, 2004). A pesar de la experimentación con nuevos cultivos y técnicas, los intentos de ocupación y la distribución "de suerte de estancias", ${ }^{4}$ el excedente producido y sus intercambios eran intermitentes.

La legislación en materia de tierras de esta década propició la radicación de agentes que se orientaron hacia la producción rural. Con ello se privilegió un patrón de asentamiento con base en la propiedad privada de las tierras, fomentando el corrimiento de la frontera productiva agraria que se pretendía combinar con el desplazamiento de la población indígena ${ }^{5}$ y la expansión del dominio territorial del novel Estado argentino. Parte de ello explica la primacía del ganado lanar en el lugar (de mayor dificultad para el robo) y la decisión arquitectónica de la casa azotea en la campaña (Bayon y Pupio, 2003).

Entre 1863 y 1868 se instaló espontáneamente entre las cuencas del río Sauce Grande y el arroyo Napostá una colonia de origen británico, contabilizando hacia finales de la década un total de treinta familias. ${ }^{6}$ Este primer momento de intervención del capital británico se caracterizó, entonces, por las inversiones en estancias. Por la ubicación en una zona alejada y desfavorecida en términos de comunicaciones, probablemente los recién llegados no disponían de una trayectoria previa para amalgamarse a las redes de connacionales que se habían instalado en territorios con mayor y mejor estructura para la producción agropecuaria. Más allá de ello y una vez instalados, se articularon nuevas redes en el sur bonaerense, en las cuales no solo transitaron personas sino también recursos materiales, gerenciales, relacionales y saberes (Fernández, 2004).

El asentamiento evidenció un proceso de capitalización orientado a la producción rural en la región. Para 1876 la colonia ocupaba 18 leguas de campo y se habían invertido en ella 14100 libras. $^{7}$ De los 79 extranjeros que habían arribado, 49 habían regresado a sus lugares de origen, 9 murieron por diferentes causas quedando un total de 21 colonos (Cernadas, 1992). Además de los peones ingleses también se había contratado personal local, pero esta mano de obra era considerada escasa y cara (Mulhall y Mulhall, 1869). Los llamados indios amigos de la zona, es decir colaboradores del gobierno, aunque con alianzas lábiles y temporales (Ratto, 2007), actuaban como braceros en los periodos de cosechas (Caronti, Real de Azua y Laspiur, 1869). Parte de los peones ingleses contaban con herramientas y animales ${ }^{8}$ lo que podría indicar que las tierras estuvieran bajo un sistema de arriendo o similar, explicando este hecho el rechazo

\footnotetext{
${ }^{4}$ En estos términos se expedía el decreto del 28 de julio de 1864 que reglamentaba la distribución de las tierras en los partidos de Bahía Blanca y Patagones (Goyena, 1892; Valencia, 2001).

${ }^{5}$ No debemos suponer que las poblaciones indígenas carecían del conocimiento relativo y de la ausencia de prácticas agrícolas (véase Jiménez y Alioto, 2007) sino que sus pautas económicas, aunque con interrelaciones con la sociedad criolla, antagonizaban crecientemente con los intereses y la orientación económica de los sectores dominantes nacionales en expansión (Villar, 2012).

${ }^{6}$ Algunos de los colonos eran Backenbury, Broadbend, H. L. Black, F. Cabbold y M. Cattery, Cheeke, Dobson, Donner, Enrique Edwards, Fewcas, Goodhall, Holmes, Hutchinson, Arturo y Juan Milred, Lane, Linwood, Maclachlan, Nicholson, Parker, Rushton, Juan Sinclair, Bryan Smith, C. y G. Shuttle C., Walker, Wood (Mulhall y Mulhall, 1869).

${ }^{7}$ Un reporte del mismo año constaba que se habían construido casas y galpones de material, ranchos y establecimientos como herrerías y molinos de vapor. Contaban, asimismo, con 12000 ovejas, algunos bueyes, vacas lecheras y caballos.

${ }^{8}$ El caso de C. y G. Shuttle y C. J. Hutchinson.
} 
de varios de ellos a fraccionarlas. La cantidad de animales que poseían destacaban entre los establecimientos de la región, según crónicas de la época. Sus conocimientos sobre las actividades rurales les permitían ensayar los cultivos "a gran escala" (Caronti et al, 1869) como también el material tecnológico con el que contaban. ${ }^{9}$ Justamente, los saberes trasladados estaban referidos al cultivo de cereales (trigo, cebada, papas y remolachas) y animales (ovejas y caballar) (Mulhall y Mulhall, 1869).

Según los protagonistas, la fertilidad de los suelos no era lo esperado, lo cual justificaba la extensión de los campos y la necesidad de mano de obra para trabajarlo. Los contactos entre esta colonia e Inglaterra fueron asiduos, especialmente con la intención de buscar parientes, amigos y peones, como también con el fin de traer maquinaria para labrar los suelos (Rojas, 1984). También enviaron muestras de la tierra a la Real Sociedad de Horticultura en aquel continente. En 1872 el periódico inglés Graphic relató la presencia en la metrópoli de uno de ellos: Arturo Mildred. Su viaje tenía el propósito de buscar tecnología y mano de obra para llevar a las tierras americanas (Míguez, 1985).

Nuevas formas de administración y organización se implementaron en la colonia dando inicio a sociedades anónimas. De ello resultó el estrechamiento de lazos y el aumento de las tierras puestas en producción como también los capitales. ${ }^{10}$ Estas formas asociativas novedosas auguraban, a su vez, la diversificación de las actividades entre los miembros. De ello derivó que, por ejemplo, Edmundo P. Goodhall se radicara temporalmente en Bahía Blanca y sirviera como intermediario para la comercialización de los productos y el desarrollo de la colonia. ${ }^{11} \mathrm{El}$ espacio para la comercialización de estos productos fue Buenos Aires, aunque la cercanía al poblado de Bahía Blanca que se iba convirtiendo en un nodo relevante del comercio regional, hizo que parte de los mismos sean remitidos allí. De este modo, se inició la presencia de intereses británicos en esta plaza -el mismo Goodhall es ejemplo de ello-, cuestión que permanecería en las sucesivas décadas mutando sus formas e impactando no solo en el terreno económico, sino también en el plano simbólico (Ribas, 2008).

El impulso productivo dado por los ingleses hizo que para 1869 la preeminencia en los cultivos del partido sean el trigo y la cebada, llevados a cabo principalmente en la colonia (Caronti et al., 1869) contando con 24 cuadras de tierra sembradas (Mulhall y Mulhall, 1869) gracias a la tecnología que posibilitaba practicarlo en grandes extensiones. Esta producción se combinó con la cría de ovinos y ganado caballar, redundando en inversión infraestructural acorde.

La cuestión de la propiedad de las tierras se presentó como un reclamo recurrente por parte de los colonos, que las habían recibido en enfiteusis y querían asegurarse su posesión solicitándolas al gobierno provincial en dos oportunidades en 1875 y 1876. Finalmente, por una ley del 30 de octubre de 1876 el Estado provincial autorizó la venta de los terrenos de la colonia inglesa a sus integrantes, consolidando las posiciones de varios de ellos (Goyena, 1892). Empero, hacia fines de la década de los setenta, la gran mayoría no las adquirió o las había vendidos y migrado a otros lugares de la provincia

\footnotetext{
${ }^{9}$ Específicamente poseían ocho arados Howard, una cosechadora, cuatro gradas, tres carretas para bueyes, una máquina de vapor y tres segadoras (Rojas, 1984).

${ }^{10}$ Goodhall y Mildred Cía. se habían asociado como los primos Enrique Edwards y Juan Walker.

${ }^{11}$ Así lo demuestra un conjunto de cartas intercambiadas con los demás habitantes como Lachlan, Walker, Catty, Black, Sinclair, solicitándole que realice depósitos de dinero, facilite elementos para realizar manufacturas y/o diligencias en general, para el desarrollo de la colonia en Sauce Grande (Rojas, 1984).
} 
como fue el caso de Walker y Edwards. Del núcleo inicial, fue Edmundo P. Goodhall quien permaneció en la región. Su estancia, ubicada en Paso Mayor, contaba con diversas plantaciones y cría de animales. Además de sus actividades rurales, profundizó sus actividades comercializadoras y se desempeñó como agente e intermediario de un conjunto de empresas británicas en un nuevo contexto que se abrió con la llegada de las empresas de transporte también del mismo origen.

Según informes de hacendados de la época se visualizaban dos problemas que afectaban a la colonia en particular y a la expansión de la frontera agraria en general: la inseguridad derivada de la presencia indígena y la falta de trasportes (Caronti et al., 1869). La respuesta a la primera demanda fue llevada adelante por el gobierno nacional a través de una serie de estrategias que buscaban controlar el territorio: fortines, distribución de tierras y finalmente la avanzada armada con la campaña dirigida por Julio A. Roca en 1879 que exterminó y desplazó a la población nativa. Este hecho anuló aquellos pactos y acuerdos con los cuales se habían formado redes comerciales y las vinculaciones en la zona sur y concluyó con "la existencia autónoma de las sociedades indígenas de las pampas y del norte patagónico" (Villar, 2012, p. 269). Asimismo creó las condiciones para acelerar la conformación de un mercado de tierras para su compra y venta en régimen de propiedad privada (Pupio y Perrière, 2013). A partir de allí en la región se sentaron las bases para la definitiva ampliación de la frontera agraria moderna, aunque perduraron los conflictos en torno a la extracción de recursos entre diversos grupos dentro de un régimen de propiedad privada y libre mercado, y bajo la égida del Estado en sus diversas administraciones que pasó a desempeñar un rol clave en la génesis de este campo de poder.

\section{Un segundo modo de intervención: Las compañías legalmente autónomas}

Si bien el proceso de capitalización de los establecimientos rurales continuó, en esta segunda etapa su desarrollo fue simultáneo a la radicación de las compañías legalmente autónomas británicas. ${ }^{12}$ Principalmente materializadas en la región por las empresas de transporte férreo, caracterizaron otro modo de ingreso del capital británico para el fomento de una producción agrícola a gran escala con destino al mercado nacional y externo. No obstante, contrariamente al periodo anterior, no estuvieron directamente vinculadas a la producción agraria sino que se valieron del impulso de la expansión de transportes y comunicación en la campaña, de recursos relacionales entre los sectores locales con el fin de reproducir sus posesiones y beneficios y de la presencia de un conjunto de agentes intermediarios ubicados en la plaza bahiense.

En virtud del accionar de estas compañías, el desarrollo urbano/rural y el capital británico quedaron estrechamente vinculados ya que los modernos medios de comunicación con la capital y el exterior (tanto el ferrocarril como el puerto) que dinamizaban la producción regional pasaron a manos del Gran Ferrocarril del Sud (en

\footnotetext{
12 Para un análisis de las mismas puede consultarse a Wilkins (1988). Un abordaje de caso sobre su desempeño en Argentina se encuentra en Lanciotti (2011).
} 
adelante FCS) ${ }^{13}$ y de Bahía Blanca and North Western Railway (en adelante FCBBNO) ${ }^{14}$ desde 1884 y 1891 respectivamente. Esta nueva incorporación tecnológica propició el aceleramiento y la transformación de las fuerzas productivas orientadas a la extracción de los productos del agro: su impacto a nivel productivo se entiende por el rol clave que desempeñaron en los diferentes sectores de la cadena de producción, generando una serie de eslabonamientos hacia la producción agraria. ${ }^{15} \mathrm{La}$ articulación de circuitos de comercialización agrario-urbanos se hizo permanente y sistemática, especialmente por la posesión de ventajas absolutas entre transporte de campaña (ferrocarril) y muelle por parte del FCs. De cualquier manera, en tanto las empresas de transporte no controlaban directamente los mecanismos de crédito agrario y la comercialización interna (desde los productores a los exportadores o los consumidores finales) que eran llevadas a cabo por agentes intermediarios, consignatarios y propietarios de barracas, las vinculaciones entre estos sectores y las empresas se hizo necesaria y mutó desde la cooperación al antagonismo según las diversas coyunturas.

El arribo progresivo de una serie de firmas y mercados, cuyos beneficios redundaban en la expansión de las actividades agrarias y sus tareas de comercialización, acompañó la instalación del ferrocarril. Para su localización aprovecharon los activos intangibles (especialmente conocimientos y capacidades vinculares y directivas) de los agentes locales, privilegiando aquellos que eran naturales británicos (Lluch y Lanciotti, 2012), como también lo aprovecharon estos para posicionarse en la plaza. Este accionar fue característico de las compañías británicas que se hicieron de las redes sociales anteriores para reforzar sus intereses en estos territorios fronterizos. Ejemplo de ello fue Edmundo P. Goodhall quien, como antiguo poblador de la colonia inglesa, tenía una trayectoria en la economía local que utilizó para ubicarse como agente de empresas y mercados ingleses como: Lloyd's Londres -mercado de seguros británicos-, de la línea de vapores Lamport \& Holt, del Banco de Londres -especialmente en la venta de letras de cambio- y del mismo FCS. Su casa comercial, asimismo, desempeñaba funciones prestamistas (Goodhall, 21 de noviembre de 1891). Carlos C. Cumming, por su parte, actuó como corredor marítimo, comisionista, consignatario y agente en general focalizándose especialmente en la introducción de maquinarias agrícolas (Guía comercial de Bahía Blanca, 1897).

El Estado, tanto nacional como provincial, a partir de las sucesivas políticas que concedían privilegios y habilitaciones, ayudó a la interrelación entre empresas inglesas, desarrollo agrario y comercializadores locales. En 1881 ante una coyuntura de posible expropiación por parte del gobierno provincial, el FCS acordó extender sus líneas hacia el sur teniendo su fin en el puerto de Bahía Blanca (Stone, 1993). El

\footnotetext{
${ }^{13}$ FCS fue una sociedad anónima creada en Londres en 1862 que se hizo de las concesiones gestionadas por Eduardo Lumb de una línea férrea hacia Chascomús, con el fin de explotar el auge lanar. Al comienzo contó con 198 accionistas y con la ayuda de los banqueros Baring Brothers y David Robertson (Rögind, 1937).

${ }^{14}$ FCBBNo fue constituida en Londres en 1889, adquirió la concesión del ramal proyectado por la compañía de D’Abreu y Torres que unían Bahía Blanca con Villa Mercedes, San Luis. En el año 1896 esta firma fue autorizada a construir un muelle propio en el estuario de Bahía Blanca el cual se inauguró en 1905, tomando el nombre de "Puerto Galván".

${ }^{15}$ Por medio de distribución de primas y beneficios se incentivó a los diferentes actores para que invirtieran o realizaran desarrollos en el agro. Frente a la gran invasión de la langosta de 1891, por ejemplo, el FCS otorgó premios y cargas gratuitas para aquellos productores que aplicaran medidas con el fin de combatir esta plaga que hacía peligrar los cultivos (FCS, 27 de noviembre de 1891).
} 
trazado férreo elegido para unir este punto con Azul respondió a la presión de los hacendados instalados en la región propiciando el aumento del valor de sus campos (Ribas, 2008).

Esta negociación le permitió al FCs extender sus beneficios sobre el sur bonaerense, sumado a los que ya gozaba en otros puntos de la provincia; por un decreto del 29 de enero de 1883 se le concedió la construcción de un muelle exclusivo en el estuario bahiense para trasladar los materiales. Si bien la gestión quiso ampliar estos privilegios para los demás artículos no lo logró en un primer momento. No obstante, en julio obtuvo una prorroga hasta 1902 para la construcción y explotación del puerto, corroborando su monopolio absoluto sobre la franja costera (Lázzaro, 1992). De este modo, la infraestructura necesaria para el desarrollo de las fuerzas productivas y para las vinculaciones con el comercio exterior quedó en manos de la iniciativa privada detentando ventajas competitivas exclusivas (de tipo monopólicas) (Lluch y Lanciotti, 2012).

Mientras el FCS ubicó su dependencia de operaciones de la zona sur en el poblado de Bahía Blanca, el fabBNo la convirtió en el inicio de su recorrido y su centro administrativo. A causa de su condición portuaria, la ciudad se formuló en el centro de un área de influencia que se fue ampliando en función de la extensión del tendido férreo y de las estaciones inauguradas. ${ }^{16}$ Se conformó así una compleja red de estaciones que articularon la zona sur, fomentando la producción y la radicación de colonias en ciertos territorios (Grippo, 1998). El FCs estuvo orientado a unir el sur provincial. ${ }^{17}$ Hacia 1897 comenzaron las ampliaciones del ramal hacia Neuquén, aumentando la zona de influencia del puerto de Bahía Blanca y restructurando, como veremos más adelante, la comercialización en esta plaza. El FCBBNo, por su parte, privilegió el oeste bonaerense y el contacto con el territorio pampeano ${ }^{18}$ (Figura 1 ).

Las nuevas configuraciones territoriales y la explosión demográfica y productiva -propiciada por la actuación de las empresas de transporte-favorecieron en el mediano plazo una orientación de la región hacia la producción agrícola. La transformación en las áreas puestas en producción da cuenta de ello. Si tomamos la evolución de los cultivos de trigo entre los años 1881 y 1895 , estos registran un aumento exponencial de 10 a 4525 hectáreas respectivamente. Asimismo los ganados muestran un aumento absoluto de cabezas, aunque disminuyeron relativamente entre 1884 y 1895 . El lanar fue el preminente y en 1895 advertimos la presencia de vacunos refinados que no fueron registrados en 1881. El gran incremento de las hectáreas cultivadas con alfalfa se encuentra vinculado a ello, revelando un cambio de pasturas que traería en los siguientes años un nuevo direccionamiento productivo a la cría de bovinos (Barsky, 1988). El desarrollo tecnológico indica esta evolución agrícola: en 1881 se detectaron 74 arados simples, 42 rastrillos y ninguna máquina segadora a vapor, en

\footnotetext{
${ }^{16}$ En 1894 el superintendente de tráfico del FCs de Bahía Blanca, Arturo Coleman, afirmaba que desde la dependencia de aquella ciudad se controlaba e tráfico desde La Gama (Lamadrid) hasta Tandil (Coleman, 1949).

${ }_{17}$ Con las siguientes estaciones: La Colina, Coronel Suarez, Curumalán, Arroyo Corto, Pigüé, Saavedra, Dufaur, Tornquist, Napostá, La Vitícola. En 1891 esta línea unió Bahía Blanca/Tres Arroyos, inaugurando las siguientes estaciones: Micaela Cascallares, Irene, Aparicio, Coronel Dorrego, San Román y Bajo Hondo (Guerreiro, 2011).

${ }^{18}$ Las estaciones construidas fueron: Villa Olga, Nueva Roma, Berraondo (Bahía Blanca), San Germán, Rondeau y Villa Iris (Púan), y en la gobernación de la Pampa: Villa Alba (luego Jacinto Arauz), Bernasconi, llegando en 1897 a Toay (Guerreiro, 2011).
} 
1887, únicamente, en colonia Tornquist había 12 segadoras y 86 arados, entre otros. Del mismo modo, las colonias de Arroyo Corto y Pigüé contaban con trilladoras de vapor (Censo general de la provincia de Buenos Aires, 1883; Segundo Censo de la República Argentina 1895, 1895).

Figura 1: Mapa de los ferrocarriles que convergen a Bahía Blanca

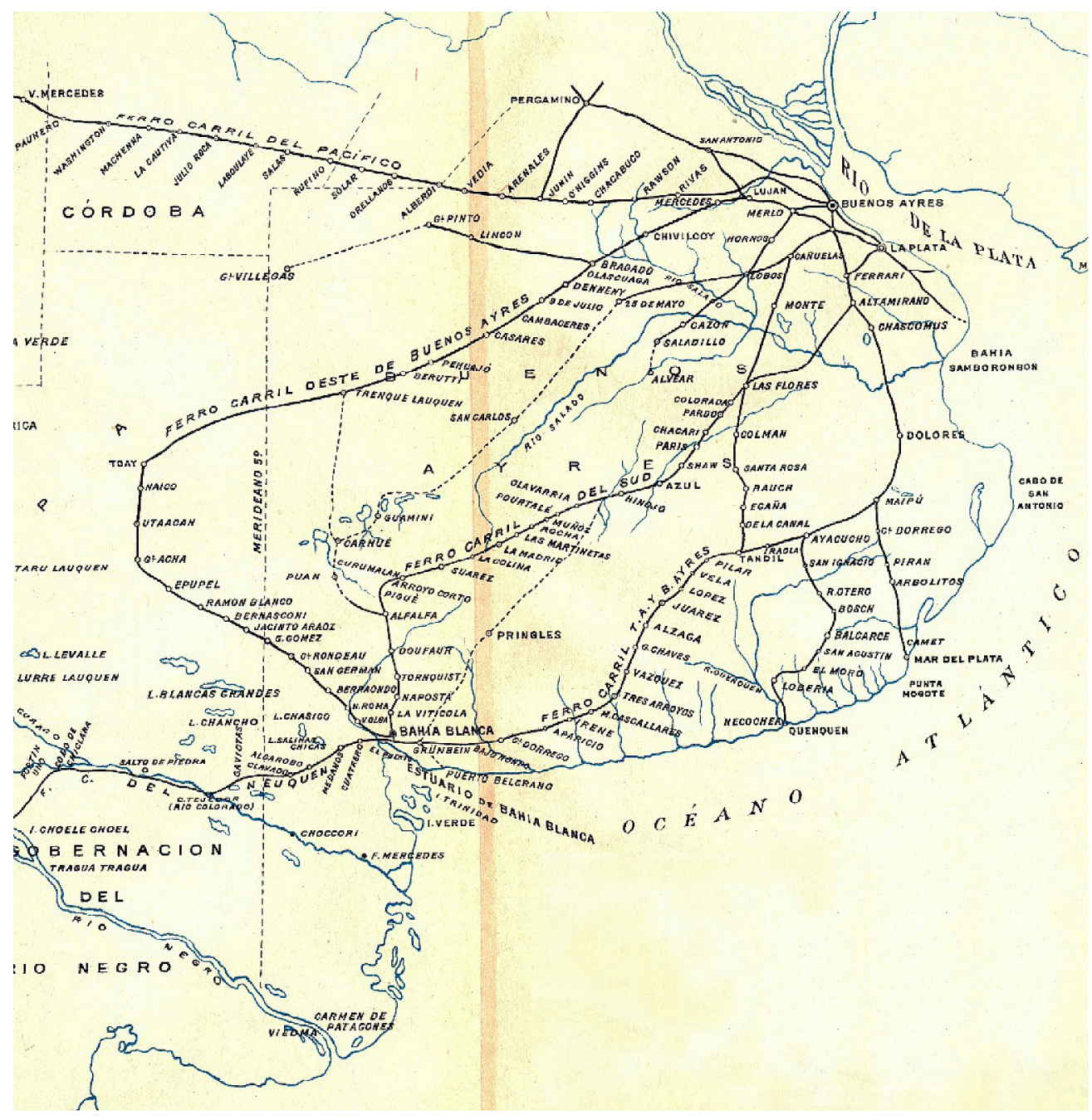

Fuente: Augusto Leone (1898).

El desarrollo de la agricultura durante las últimas décadas del siglo xIX respondió al auge colonizador de la región encarado por empresas particulares. Se fundaron Pigüé (1884), Arroyo Corto (1885) y San Martín. Entre ellas contabilizaban un total de 297 familias. Mientras que en las tierras de Ernesto Tornquist se llevaba a cabo un asentamiento de igual índole con un total de 190 personas a partir de 1883 (Morsbach, 1888). Si bien no vinculadas directamente con el capital inglés, su radicación y pervivencia fue posible por las nuevas arterias de comunicación que surcaron la región y que valorizaron la propiedad. De este modo, el paisaje registró variaciones 
que se correspondieron con la capitalización en materia de transporte. El importe de la propiedad raíz se vio duplicado o quintuplicado según su ubicación (El valor de la propiedad rural, 5 de febrero de 1886). De ello también se beneficiaron las mismas empresas ya que como parte de sus contratos, el gobierno les había concedido en propiedad terrenos en varios puntos de la región. A fines de la década de 1880 (momento de especulación en materia de tierras) el FCs proyectó la creación de La Nueva Liverpool, un poblado que iba a estar ubicado en las propiedades de la empresa en las inmediaciones del puerto. ${ }^{19}$ Más aún hacia el año 1891 el FCs alquilaba sus chacras en el Puerto de Bahía Blanca a un "precio módico", encargado de ello era el inspector de tráfico local (Ferrocarril del Sud, 25 de octubre de 1891).

\section{Las empresas británicas y sus estrategias de gestión en el plano regional}

Además de la acción estatal que la posibilitó y aseguró, la reproducción de este tipo de empresas se sostuvo a partir de sus nexos sociales en la región que fueron generados y movilizados por sus representantes que sirvieron de intermediarios. Así pues, simultáneamente con el arribo de las firmas se dio el de sus directivos británicos, inaugurando un tipo de inmigración diferente a la que analizamos anteriormente. Esta nueva corriente ya no correspondió a agricultores con capital propio, sino a empleados, dirigentes y profesionales de las diferentes empresas ferroviarias. Por esta condición su establecimiento no era permanente sino que estuvo atado a las directivas de las compañías. Si para el año 1869 en este partido contabilizamos 33 inmigrantes de esta procedencia -que simbolizaban principalmente miembros de la colonia- para 1895 este número se había elevado a 222 (Primer Censo de la República Argentina 1869, 1872; Segundo Censo de la República Argentina 1895, 1895). Aunque no cuantitativamente relevante en términos relativos, la gravitación de esta colectividad se hizo notar ya que se ubicaron en áreas claves de una economía local que tendía a la especialización agraria: transporte, comercialización y el sistema crediticio.

En relación con las empresas de transporte, las condiciones monopólicas que detentaban generaron tensiones con los sectores comercializadores internos (propietarios de barracas y consignatarios ${ }^{20}$ que se volcaron en la prensa periódica. El precio de las tarifas en el transporte y las descargas en el puerto, el izado de la bandera inglesa en el muelle, las cuantiosas garantías que percibía la empresa del gobierno y su deficiencia en el servicio, entre otros, fueron algunos de los reclamos elevados. ${ }^{21} \mathrm{~A}$ pesar de los fluidos contactos que unían a estos sectores, la burguesía local no dejó de inquietarse por el avance de los intereses británicos en la economía, reconociendo a la empresa del FCS como un "pulpo". Desde su radicación en la ciudad en 1884 se la había atacado a raíz del privilegio que detentaba sobre el muelle. Lo que se encontraba

\footnotetext{
19 Según El Argentino, un diario local, este plan consistió en "una de las tantas buenas especulaciones hechas por el inglés ojo-avizor que viene a nuestras playas a especular mientras nosotros los criollos, perdemos el tiempo en paradas políticas de barrio" (Nueva Liverpool, 9 de septiembre de 1887).

${ }^{20}$ Para una clasificación de los mismos se puede consultar a Crisafulli (1994).

${ }^{21}$ Puede consultarse en las siguientes noticias: Ferrocarriles (5 de marzo de 1885); No se puede silenciar (17 de abril de 1885); Nuestro Puerto (20 de noviembre de 1886).
} 
en discusión era las mayores o menores ganancias entre los diferentes sectores de la cadena de producción del agro: productores, consignatarios, propietarios de barracas y transportistas.

Esta situación exigió que las acciones gerenciales de las empresas contemplaran mecanismos de integración con la comunidad local para, por un lado, disminuir los niveles de enfrentamiento $y$, por otro, aumentar y asegurar sus ganancias. Al igual que en la capital (Lewis, 2007), en Bahía Blanca surgieron instituciones y agrupaciones anglo-criollas cuya base estaba en la red de negocios que unían a los británicos con los sectores comercializadores locales. Empero, en nuestro caso la ausencia de un pasado mercantil previo posibilitó que las empresas tuvieron un fuerte rol en la construcción de estas tramas que fueron creciendo a la luz de la estructuración de una ciudad moderna. Era la misma lógica de operación de las free standing companies -con su particular y débil estructura gerencial- y su orientación a la rama de los servicios en el plano regional que propendían a una mayor articulación con los grupos locales (Wilkins, 1988). De ello deriva que encontremos británicos formando parte de diversas organizaciones ya sean culturales como la participación en clubes sociales, bibliotecas, etc., o específicamente comerciales y agrarias. Si pensamos en los impactos e injerencias que tenían las empresas en los diferentes eslabones del agro local, advertimos que sus representantes contaban con un amplio margen de acción para participar en múltiples iniciativas que atañían al desarrollo regional.

Durante el periodo, un caso que sobresale fue el de William Harding Green quien, como gerente de tráfico del Ferrocarril Bahía Blanca Noroeste, participó del Club Argentino (1906), en la Sala Comercial (1897) y en la organización de la Sociedad Rural de Bahía Blanca (1896), siendo un activo participante en las sucesivas comisiones directivas a pesar de que no hallamos registros que poseyera ni arrendara tierras o ganados (Pérez, 1994). ${ }^{22}$ Edmundo P. Goodhall también tuvo una labor fructífera en diversas sociedades locales ya que participó en la conformación de la Asociación Rural ${ }^{23}$ y luego de la Sociedad Rural de Bahía Blanca, del Banco de Crédito Real y Personal y en sus sucesivos directorios (1889) -organización local que buscaba fomentar la producción regional- asimismo, fue vicecónsul británico entre 1882 y 1891 y tuvo un breve desempeño como concejal en el año 1886 como miembro de las fuerzas políticas aliadas a Dardo Rocha, desligándose luego de la política partidaria.

De este modo, los británicos fueron amalgamándose en las esferas de sociabilidad política, productiva y cultural de la ciudad ubicándose como intermediarios entre empresas y diferentes grupos vinculados al agro que iban desde comercializadores hasta productores. Incluso, la falta de una asociación sectorial de extranjeros de esta nacionalidad al igual que su ausencia en la creación del Centro Político Extranjero -impulsado a mediados de 1891 por la aprobación de la nueva ley Orgánica de Municipalidades-, corrobora la visión de una comunidad británica más bien orientada

\footnotetext{
${ }^{22}$ Su genealogía, asimismo, da cuenta de las redes sobre las cuales se basaba la elección de personal gerencial de las empresas británicas ya que William era hijo de John Green primer gerente del Banco de Londres y el Río de la Plata. Su orientación hacia la rama de los transportes inició tempranamente cuando comenzó a trabajar en el Great Eastern Railway de Inglaterra para continuar en 1878 en el Ferrocarril Central Uruguayo. Durante la década de los ochenta trabajó en el cuerpo de ingenieros del FCs en Argentina y en 1891 fue nombrado como director del FCBBNo en Bahía Blanca, cargo que conservó hasta 1924.

${ }^{23}$ Esta organización fue un primer intento radicado en la ciudad de Bahía Blanca de conformar una sociedad rural regional, sin embargo por desavenencias políticas no funcionó (Asociación Rural de Bahía Blanca, 15 de julio de 1886).
} 
a hacer prevalecer sus intereses por otros medios (Caviglia, 1984). De esta manera se privilegiaba la intervención a través del desempeño individual y de medios no necesariamente partidarios.

Es así como se fueron cimentando relaciones fluidas de dependencia con los comerciantes locales que serían canalizadas para obtener mayores beneficios y alianzas a la hora de la conflictividad social y obrera. ${ }^{24}$ A cambio, las empresas eran las poseedoras del capital necesario para realizar obras de infraestructura y servicios con el fin de impulsar la producción agropecuaria regional conjuntamente con las actividades de los comerciantes locales.

Sin embargo, los nexos de cooperación se combinaron con los conflictivos, especialmente al solaparse las funciones de estos dos grupos, lo cual sucedió a mediados de la década de los noventa cuando el FCBBNo inició una campaña para construir un mercado de frutos en la ciudad. En este punto encontramos nuevamente posiciones encontradas entre los comerciantes, principalmente los propietarios de barracas, quienes veían peligrar sus tareas de acopio. El gobierno local, que tenía a Jorge Moore como intendente y que respondía en mayor medida a la Unión Cívica Radical (Molina, 2007), aprobó la construcción del mismo. El elenco de este partido se encontraba fuertemente ligado al comercio y a la producción ganadera. Más aún, a través de la normativa emitida por esta dependencia sería reconocido como el punto oficial para la revisación de los frutos, reemplazando la antigua Plaza de Frutos pública. La comuna le brindaba, asimismo, la exclusividad de las actividades ya que prohibía otra construcción similar dentro del radio de su administración por el término de veinte años (Mercado central de frutos, 22 de mayo de 1896). Esta acción implicaba un avance de las firmas inglesas sobre otros eslabones de la cadena de producción y comercialización, como también hacia la dinámica urbana/comercial que fue centralizada en la ciudad de Bahía Blanca. Si bien se advertía que la obra era necesaria para posicionar la plaza como una opción a los mercados del sur, las voces opositoras demandaban su regulación y control por parte de los mismos comerciantes (Mercado central de frutos, 26 de mayo de 1896).

Esta tensión se aplacó por una medida que reposicionó y expandió la plaza bahiense en conjunción con los intereses de las empresas británicas: la extensión del ramal del FCs hacia Neuquén. La iniciativa incorporó nuevas tierras a la orientación atlántica-exportadora, acrecentando el influjo y monopolio de la empresa que excedió la provincia. La coyuntura conflictiva con Chile y el apremio del gobierno nacional le facilitó a la compañía negociar su construcción con considerables garantías que incluía: tierras expropiadas por nación, excepción de impuestos para los transportes de cargas, aplazamiento de la exención tributaria para importar productos que estaba próximo a vencerse (en 1902), derechos sobre el puerto de Bahía Blanca y exención de impuestos nacionales, provinciales y municipales por el término de cincuenta años, entre otros (Rögind, 1937).

De este modo, se presentaban nuevos territorios de influencia para los intermediaros presentes en la plaza de Bahía Blanca. Incluso, los espacios, ahora alcanzados por el transporte, experimentaron transformaciones que le abrieron una posibilidad atlántica a la producción, iniciando una nueva articulación entre estas empresas, el

${ }^{24}$ Cuestión que se visibilizó mayormente con las grandes huelgas que protagonizaron obreros del ferrocarril y el puerto a inicios del siglo xx (véase Caviglia, 1993; Fanduzzi, 2005; 2007; Randazzo, 2007). 
Estado y comercializadores en la expansión de la frontera productiva. ${ }^{25}$ De la plaza local podemos mencionar la ampliación de las operaciones de las sociedades Mendez y Cía, Lanusse y Olaciregui, Diego Meyer y Cía. hacia el sur (Gentile et al, 1998; Ruffini, 2007). Esto reveló, entonces, el avance en conjunto de las firmas inglesas y de las casas consignatarias bahienses, que si bien coyunturalmente conjugaban intereses comerciales, prontamente se volverían a tensionar por la necesidad de la comercialización y el reducido puerto inglés a inicios del siglo xx.

\section{Reflexiones finales}

Las nuevas dinámicas de la economía-mundo a fines del siglo xIx involucraron progresivamente las zonas marginales. Para esto se precisó la actuación de un conjunto de actores: Estado, agentes locales e internacionales que desde diferentes funciones fomentaron el corrimiento de la frontera agraria. Capitales, recursos materiales e inmateriales fueron movilizados con este fin que, en el caso de estudio, estuvieron motorizados en gran medida por el capital británico en alianza con comerciantes locales que usufructuaron la conexión de la región con los flujos financieros y comerciales exteriores.

A lo largo de este artículo analizamos las modalidades de intervención del capital inglés en el sur bonaerense, en el partido de Bahía Blanca, y sus vinculaciones con la expansión de la frontera productiva agraria orientada a la exportación, detectando dos formas que se sucedieron cronológicamente y se vincularon con una serie de actores y modos de actuación en el escenario bahiense.

La participación británica en el agro, en primer lugar, fomentó la capitalización de estancias para el cultivo del trigo a gran escala conjuntamente con la cría de ovinos y yeguarizos. Los colonos ingleses se presentaron como componente del corrimiento de la frontera agraria dado el traslado de conocimientos rurales, tecnologías y modos de administrar los campos. Mientras que el Estado provincial reguló la concesión de tierras, el nacional aplicó diferentes estrategias sobre la población indígena que culminó con la campaña dirigida por Julio A. Roca, lo que derivó en el fin de la existencia autónoma de estos pueblos. A partir de allí se generaron las condiciones para una nueva etapa de configuración y articulación del capital británico en la frontera productiva regional.

La acción de las compañías legalmente autónomas caracterizó un segundo momento. De la actuación individual o en pequeñas sociedades en el agro se pasó a la preferencia por la inversión en los servicios a través de grandes empresas que permitían, a su vez, negociar mejores condiciones y garantías con el Estado. Agentes radicados oficiaron de nexos de algunas de ellas explotando sus diversos capitales, mientras que otras trasladaron sus propios representantes que modificaron el paisaje

${ }^{25}$ Así lo relató Arturo Coleman -superintendente del FCS-:

Al llegar los primeros trenes a Neuquén y establecerse allí la gobernación, comenzaron a instalarse activamente agencias de transporte carretero, almacenes y casas de negocios de toda índole, y gradualmente la población y productos del territorio y de la cordillera fueron encauzándose más hacia los mercados argentinos. Lo mismo ocurrió con los de las Colonias Lucinda y Roca en el Alto Valle (Coleman, 1949, p. 179). 
relacional bahiense. Particularmente, el ferrocarril actuó como una infraestructura articuladora de la región, haciendo de Bahía Blanca su núcleo, por las ventajas que en el muelle detentaba el FCs. Su zona de influencia se ampliaba al calor de las extensiones de los ramales al igual que la producción cerealera y la cría de lanares primero y vacunos después. Otros fenómenos derivados de la expansión ferrocarril fueron la colonización y el aumento del valor de los terrenos por su posición relativa como también por la inversión practicada en infraestructura y servicios.

Más allá del impacto cuantitativo en el mundo rural, el éxito de las empresas radicó en sus estrategias gerenciales en materia social. Como fenómeno particular de este tipo de organización empresarial, las medidas implementadas por sus representantes estuvieron vinculadas a arraigar y pujar por sus intereses sectoriales con el fin de consolidar los privilegios logrados con los gobiernos nacionales y provinciales en la región. Como su desempeño en el plano productivo y regional residía en la articulación de actividades con otros sectores como productores y comercializadores rurales, sus estrategias conllevaron la actuación en asociaciones que buscaron organizar y complejizar el agro como las asociaciones rurales y comerciales.

En este marco, la socialización con sectores que ocupaban cargos de poder local les permitió a los representantes de las empresas británicas movilizar sus influencias en ciertos momentos. El caso demuestra alianzas coyunturales que se desplegaron en torno a la consecución de beneficios para comercializadores y empresas que a la larga fueron generando redes de dependencia pero sin soslayar momentos de tensión y conflicto. Al poseer diversas inversiones en la región, las empresas obtenían una serie de réditos de los cobros de tierras, arriendos y servicios portuarios, además de los férreos. Esto generó fases de tensión con los comerciantes locales, pero la imbricación de intereses en las mencionadas asociaciones permitió desplazar las hostilidades, como también lo hizo la extensión del ramal a Neuquén. Resultado de ello fue la consolidación de la plaza bahiense y de sus actores que fueron especializándose en las actividades de intermediación en dependencia de los activos de estas empresas.

\section{Agradecimientos}

Se agradecen los sugerentes comentarios y aportes realizados por Marcela Tejerina, Luciano Lorenzetti y Joaquín García Insausti.

\section{Referencias}

Alioto, S. (2011). Indios, frontera y ganado. La ruta del río Negro (1750-1830). Rosario: Prohistoria.

Asociación Rural de Bahía Blanca. (15 de julio de 1886). El Porteño.

Barbero, M. I. y Rocchi, F. (2004). Cultura, sociedad, economía y nuevos sujetos de la Historia: Empresarios y consumidores. En B. Braggoni (Ed.), Microanálisis. Ensayos de historiografía argentina (pp. 103-143). Buenos Aires: Prometeo.

Barsky, O. (Ed.). (1988). El desarrollo agropecuario pampeano. Buenos Aires: IDEC, INTA, IICA. 
Bayon, C. y Pupio, A. (2003). La construcción del paisaje en el sudoeste bonaerense (1865-1879): una perspectiva arqueológica. En R. Mandrini y C. Paz (Eds.), Las fronteras hispanocriollas del mundo indígena latinoamericano en los siglos XVIII-XIX. Un estudio comparativo (pp. 343-374). Bahía Blanca: IEHS, UNCPBA-CEHIR, UNCO-UNS.

Benedetti, A. y Salizzi, E. (2014). Fronteras en la construcción del territorio argentino. Cuadernos de Geografia: Revista Colombiana de Geografia, 23(2), 121-138. https:// doi.org/10.15446/rcdg.v23n2.38366

Benedetti, G. M., Andreozzi, G. B., Duval, V. S., Fernández, M. E., Gazzolo, B. I., González, P. A. ... Volonté, A. (2015). Geografías locales. Bahía blanca: del medio natural al medio construido. Bahía Blanca: Ediuns.

Bourdieu, P. (2002). Campo de poder, campo intelectual. Buenos Aires: Montressor.

Caronti, F., Real de Azua, E. y Laspiur, S. (1869). El partido de Bahía Blanca. Informe de la comisión de la exposición nacional de Córdoba por la comisión especial de aquel partido. Buenos Aires.

Caviglia, M. J. (1984). Inmigración ultramarina en Bahía Blanca. Buenos Aires: Clacso.

Caviglia, M. J. (1993). Ingeniero White: la huelga de 1907. Bahía Blanca: Cocina del Museo del Puerto de Ingeniero White.

Censo general de la provincia de Buenos Aires: demográfico, agrícola, industrial, comercial verificado el 9 de octubre de 1881 bajo la administración de Dardo Rocha. (1883). Buenos Aires: Imp. El Diario.

Cernadas, M. (1992). Inmigración en Bahía Blanca: la ciudad y la campaña en 1869. En M. Cernadas, N. Buffa y A. Eberle (Eds.), Estudios sobre inmigración (Vol. I, pp. 5-44). Bahía Blanca: Departamento de Humanidades. Universidad Nacional del Sur.

Cernadas, M., Buffa, N. y Eberle, A. (1992). Estudios sobre inmigración (Vol. I). Departamento de Humanidades. Universidad Nacional del Sur.

Costantini, F. y Heredia, E. (2018). El progreso en cuestión. Sectores productivos, políticas económicas y conflictividad social. En M. Cernadas y J. Marcilese (Eds.), Bahía Blanca siglo xx. Historia política, económica y sociocultural (pp. 153206). Bahía Blanca: Ediuns.

Crisafulli, G. (1994). Para una historia de la burguesía pampeana. Terratenientes y comerciantes en el sur bonaerense a fines del siglo xix. Estudios Sociales, IV(7), 69-81.

Coleman, A. (1949). Mi vida de ferroviario inglés en la Argentina, 1887-1949. Bahía Blanca. El valor de la propiedad rural. (5 de febrero de 1886). El Porteño.

Fanduzzi, N. (2005). ¿Cómo empirizar el tiempo? Ensayos de periodización desde la perspectiva existencial (ejemplo: trabajadores, puerto y modernización). En M. Cernadas y M. Vaquero, del C. (Eds.), Estudios culturales, modernidad y conflicto en el sudoeste bonaerense (pp. 13-21). Bahía Blanca: Universidad Nacional del Sur.

Fanduzzi, N. (2007). Algunas propuestas sobre cómo construir la historia de los trabajadores portuarios del período agro-exportador en Ingeniero White. En II Jornadas de Investigación en Humanidades. Bahía Blanca: Universidad Nacional del Sur.

FCS. (27 de noviembre de 1891). El Porteño.

Fernández, A. (2004). Relaciones entre flujos comerciales externos y movimientos migratorios: lo que puede demostrar el cambio de escala en un estudio de caso. En B. Bragoni (Ed.), Microanálisis. Ensayos de historiografía argentina (pp. 81-102). Buenos Aires: Prometeo. 
Ferns, H. S. (1966). Gran Bretaña y Argentina en el siglo XIX. Buenos Aires: Solar.

Ferrocarril del Sud. (25 de octubre de 1891). La Tribuna.

Ferrocarriles. (5 de marzo de 1885). El Eco de la Bahía Blanca.

Gentile, B., Destéffaniz, C., Debener, M., Debattista, S. y Bertello, C. (1998). Del Pacífico al Atlántico: el intercambio comercial neuquino en las primeras décadas del siglo xx. Anuario IEHS, (13), 369-390.

Goodhall. (21 de noviembre de 1891). El Porteño.

Goyena, J. (Ed.). (1892). Digesto rural y agrario: recopilación de leyes, decretos, resoluciones, fallos de las Cortes federal y provinciales. Buenos Aires: Juan A. Alsina.

Grippo, S. B. (1998). Redes ferroviarias y redefinición de nuevos territorios en el orden mundial de fines del siglo XIx. En M. Cernadas y R. Bustos (Eds.), Estudios regionales interdisciplinarios (pp. 123-132). Buenos Aires: Universidad Nacional del Sur.

Guerreiro, H. (2011). Los ferrocarriles en Bahía Blanca (Vols. 1-2). Bahía Blanca.

Guía comercial de Bahía Blanca. (1897). Bahía Blanca: Rouquanud \& Co.

Harvey, D. (2007). Espacios del capital. Hacia una geografía crítica. Madrid: Akal.

Iglesias, E. (1968). La inmigración en Bahía Blanca 1880-1900. Revista de la Junta de Estudios Históricos de Bahía Blanca, II(1), 1-13.

Jiménez, J. F. y Alioto, S. (2007). «Que ningún desgraciado muera de hambre»: agricultura, reciprocidad y reelaboración de identidades entre los ranqueles en la década de 1840. Mundo Agrario, 8(15). Recuperado de http://hdl.handle. net/10915/13289

Kulfas, M., Porta, F. y Ramos, A. (2002). Inversión extranjera y empresas transnacionales en la economía argentina. Chile: CEPAL.

Lanciotti, N. (2011). Inversión británica y redes empresariales : la estructura organizativa y las estrategias de gestión del grupo River Plate Trust, Loan \& Agency en Argentina, 1881-1962. Anuario del Centro de Estudios Económicos de la Empresa y el Desarrollo, 3(3), 85-126.

Lanciotti, N. y Lluch, A. (2009). Gran Bretaña y Argentina: Inversiones, empresas y relaciones económicas (1870-1975c.). Balance historiográfico y agenda de investigación (Documento de Trabajo). Departamento de Humanidades. Recuperado de http://repositorio.udesa.edu.ar/jspui/handle/10908/467

Lanciotti, N. y Lluch, A. (2011). The rise and "decline" of European companies in Argentina (1870-1945c): new evidences and discussion. En 15th Annual Conference of the European Business History Association. Atena.

Lanciotti, N. y Lluch, A. (2018a). Las empresas extranjeras en Argentina desde el siglo XIX al siglo XXI. Buenos Aires: Imago mundi.

Lanciotti, N. y Lluch, A. (2018b). Staffing policies and human resource management in Argentina: American and British firms (1890-1930s). Business History, 1-25. doi: 10.1080/00076791.2018.1471061

Lázzaro, S. (1992). Estado, capital extranjero y sistema portuario argentino (Vols. 1-2). Buenos Aires: Centro editor de América Latina.

Leone, A. (1898). Plano topográfico de la ciudad de Bahía Blanca con sujeción a mensuras y estudio de títulos y datos estadísticos sobre la misma ciudad. Buenos Aires: J. Peuser. 
Lewis, C. W. (2007). «Anglo-Criollo» rather than British: early investments in Argentinian railways and utilities. En J. Schvarzer, A. Regalsky y T. Gomez (Comps.), Estudios sobre la historia de los ferrocarriles argentinos (1857-1940) (pp. 223-270). Buenos Aires: UBA.

Lluch, A. y Barbero, M. I. (2015). Historia de empresas en América Latina: breves reflexiones sobre su desarrollo y la agenda de temas de investigación en el siglo xxi. Contribuciones Científicas y Tecnológicas, 40(140), 31-38.

Lluch, A. y Lanciotti, N. (2012). Las empresas europeas en Argentina: condicionantes, destinos de inversión y cambios organizativos entre la primera y la segunda guerra mundial. Desarrollo Económico, 52(205), 119-146.

Mandrini, R. (2003). Hacer historia indígena: el desafío de los historiadores. En R. Mandrini y C. Paz (Eds.), Las fronteras hispanocriollas del mundo indígena latinoamericano en los siglos XVIII y XIX. Un estudio comparativo (pp. 13-32). Tandil: IEHS,UNCPBA-CEHIR,UNCO-UNS.

Marichal, C. (1995). Las inversiones extranjeras en América Latina, 1850-1930: nuevos debates y problemas en historia económica comparada. México: Fondo de Cultura Económica.

Martorell, G. (1969). Las inversiones extranjeras en la Argentina. Buenos Aires: Galerna.

Mercado central de frutos. (22 de mayo de 1896). El Porteño.

Mercado central de frutos. (26 de mayo de 1896). El Porteño.

Míguez, E. (1985). Las tierras de los ingleses en la Argentina 1870-1914. Buenos Aires: Editorial del Belgrano.

Miller, R. (2015). Writing the history of British business in Latin America. Contribuciones Cientificas y Tecnológicas, 40(140), 39-49.

Miravalles, A. (2013). Los talleres invisibles. Una historia de los Talleres Ferroviarios Bahía Blanca Noroeste. Bahía Blanca: Ferrowhite.

Molina, H. (2007). 1886-2003 Intendentes de Bahía Blanca. Comisionaturas. Bahía Blanca: Imprenta Fiore.

Monacci, G. (1979). La colectividad británica en Bahía Blanca. Bahía Blanca: Universidad Nacional del Sur.

Morsbach, T. (1888). Estudios económicos sobre el sud de la provincia de Buenos Aires. Buenos Aires: Peuser.

Mulhall, E. T. y Mulhall, M. G. (1869). Handbook of the River Plate. Buenos Aires: The Standard, 1869.

No se puede silenciar. (17 de abril de 1885). El Reporter. Periódico Noticioso Comercial y Literario.

Nuestro puerto. (20 de noviembre de 1886). El Argentino.

Nueva Liverpool. (9 de septiembre de 1887). El Argentino.

Pérez, E. (1994). Los cien años de la Sociedad Rural de Bahía Blanca. Bahía Blanca: Harris. Primer Censo de la República Argentina 1869. (1872). Buenos Aires.

Pupio, A. y Perrière, H. (2013). Malones, fortines y estancias en la identidad de general Daniel Cerri. Bahía Blanca: Ediuns.

Randazzo, F. (2007). Las grietas del relato histórico: apuntes sobre los orígenes del anarquismo en Bahía Blanca y la matanza de obreros de Ingeniero White en 1907. Buenos Aires: cCC Centro Cultural de la Cooperación Floreal Gorini. 
Ratto, S. (2007). Indios y cristianos. Entre la guerra y la paz en las fronteras. Buenos Aires: Sudamericana.

Ratto, S. (2008). Allá lejos y hace tiempo. El fuerte de Carmen de Patagones en la primera mitad del siglo xix. Quinto Sol, (12), 45-72.

Ratto, S. y Santilli, D. (2004). De factoría a poblado agropecuario. La evolución del partido de Bahía Blanca hacia 1869. Cuadernos del Sur Historia, (33).

Reboratti, C. (1990). Fronteras agrarias en América Latina. Geo crítica. Cuadernos Críticos de Geografía Humana, xv(87).

Regalsky, A. (1986). Las inversiones extranjeras en la Argentina (1860-1914). Buenos Aires: Centro Editor de América Latina.

Regalsky, A. (1999). Exportaciones de capital y grupos inversores: las inversiones francesas en la Argentina, 1880-1914. Anuario de Estudios Americanos, 56(2), 553-580.

Reguera, A. (2009). Empresarios de ayer, de hoy y de siempre. Un recorrido por sus formas espacio-temporales. América Latina en la Historia Económica, 32, 9-28.

Ribas, D. (2008). Del fuerte a la ciudad moderna: Imagen y autoimagen de Bahía Blanca (Tesis Doctoral). Universidad Nacional del Sur, Bahía Blanca.

Rögind, W. (1937). Historia del ferrocarril sud. Buenos Aires: Establecimiento Gráfico Argentino.

Rojas, J. L. (1984). El malón de 1870 a Bahía Blanca y la colonia de Sauce Grande. Buenos Aires: Ediciones Culturales Argentinas.

Ruffini, M. (2007). La pervivencia de la República posible en los territorios nacionales. Poder y ciudadanía en Río Negro. Bernal: Universidad Nacional de Quilmes.

Sábato, J. (1991). La clase dominante en la argentina moderna formación y características. Buenos Aires: Imago Mundi.

Salizzi, E. (2017). Notas para el estudio de la frontera agraria en Geografía: de la frontera del agricultor a la frontera agraria moderna. En S. Braticevic, C. Tommei, y Al. Rascovan (Comps.), Bordes, límites, frentes e interfaces. Algunos aportes sobre la cuestión de las fronteras (pp. 186-208). Recuperado de https://www.academia. edu/37591404/BORDES_L\%C3\%8DMITES_FRENTES_E_INTERFACES_Algunos_aportes_sobre_la_cuesti\%C3\%B3n_de_las_fronteras

Schiavoni, G. (1997). Las regiones sin historia: apuntes para una sociología de la frontera. Revista Paraguaya de Sociología, 261-301.

Schvarzer, J., Regalsky, A. y Gomez, T. (Eds.). (2007). Estudios sobre la Historia de los Ferrocarriles-Argentinos (1857-1940). Buenos Aires: UBA.

Segundo Censo de la República Argentina 1895. (1895). Buenos Aires: Talleres Tipográficos de la Penitenciaría Nacional.

Silva, H. (1987). El transporte cerealero en la región de Bahía Blanca. Auge y decadencia del monopolio ferroviario. Buenos Aires: Academia Nacional de la Historia.

Sourrouille, J., Gatto, F. y Kosacoff, B. (1984). Inversiones extranjeras en América Latina. Política económica, decisiones de inversión y comportamiento económico de las filiales. Buenos Aires: Banco Interamericano de Desarrollo.

Stone, H. R. (1993). British railways in Argentina 1860-1948. Kent: Waters.

Valencia, M. (2001). Las tierras públicas de Buenos Aires: políticas y realidades en la segunda mitad del siglo xix. Anuario del Centro de Estudios Históricos «Prof. Carlos S. A. Segreti», 1(11), 113-128. 
Vázquez, B. y Dalla, G. (Eds.). (2005). Empresarios y empresas en América Latina (siglos XVIII-XX). Maracaibo: Universidad del Zuldia.

Viego, V. (2004). El desarrollo industrial en los territorios periféricos. El caso de Bahía Blanca. Bahía Blanca: Ediuns.

Villar, D. (2012). Las poblaciones indígenas, desde la invasión española hasta nuestros días. En H. Otero (Dir.), Población, ambiente y territorio (pp. 249-278). Buenos Aires: Edhasa.

Villar, D. y Ratto, S. (2004). Comercio, ganado y tierras en la frontera de Bahía Blanca, 18501870. Bahía Blanca: Centro de Documentación Patagónica. Departamento de Humanidades.

Wilkins, M. (1988). The free-standing company, 1870-1914: an important type of British foreign direct investment. Economic History Review, XLI(2), 259-282.

Florencia Costantini

Argentina. Licenciada en Historia por la Universidad Nacional del Sur (Argentina). Actualmente es becaria doctoral del Consejo Nacional de Investigaciones Científicas y Técnicas (CONICET) y doctoranda en la Universidad Nacional del Sur del Doctorado en Historia. Líneas de investigación: políticas agrarias en clave regional hacia fines del siglo xIx y principios del xx. Publicación reciente: Constantini, F. y Heredia, E. (2018). El progreso en cuestión, sectores productivos, políticas económicas y conflictividad social. En M. Cernada y J. Marcilese (Eds.), Bahía Blanca siglo Xx. Historia política, económica y sociocultural (pp. 153-206). Bahía Blanca: Ediuns. 